\title{
La enseñanza de la meteorología en el Instituto Pedagógico de Caracas: experiencia de tres décadas
}

\author{
THE TEACHING OF METEOROLOGY IN THE INSTITUTE PEDAGOGICAL DE CARACAS: \\ EXPERIENCE OF THREE DECADES \\ Sergio Foghin-Pillin sfoghin@hotmail.com \\ Código ORCID: 0000-0003-3690-8195 \\ Universidad Pedagógica Experimental Libertador, Venezuela
}

Recibido abril 2019 | Arbitrado mayo 2019 | Publicado julio 2019

\begin{abstract}
Resumen
Palabras clave:

Venezuela;

meteorología;

enseñanza; educación

Aunque desde la creación, en 1946, del Departamento de Geografía e Historia, se incluyeron contenidos de meteorología y climatología en los programas de algunas asignaturas, el primer curso dedicado exclusivamente a estos temas se introdujo en 1969, cuando se instaura el régimen semestral en el Instituto Pedagógico de Caracas (IPC), el cual a partir de 1983 se integró a la Universidad Pedagógica Experimental Libertador. Dicho curso se ofreció también para los estudiantes del Departamento de Ciencias de la Tierra, tras su creación en 1971. A partir de 1988, en este último Departamento se crean tres nuevos cursos dedicados a las ciencias atmosféricas. Este trabajo, de carácter retrospectivo, tuvo como propósito formular algunas consideraciones y reflexiones sobre la evolución de la enseñanza de las ciencias meteorológicas en el IPC a lo largo de la carrera docente e investigativa del autor, tras una experiencia de treinta años en la mencionada institución. Se concluye con la pretensión de coadyuvar en la formación de los futuros docentes de Educación Ambiental ante los desafíos dinámicos del hoy.
\end{abstract}

\section{Abstract}

\section{Keywords:}

Venezuela; Instituto Pedagógico de Caracas; meteorology; teaching; education
Although since the creation in 1946 of the Department of Geography and History, meteorology and climatology content was included in the programs of some subjects, the first course dedicated exclusively to these subjects was introduced in 1969, when the semester regime was established in the Pedagogical Institute of Caracas (IPC), which from 1983 was integrated to the Libertador Experimental Pedagogical University. This course was also offered to students of the Department of Earth Sciences, after its creation in 1971. Starting in 1988, three new courses dedicated to atmospheric sciences were created in the latter Department. The purpose of this retrospective work was to formulate some considerations and reflections on the evolution of meteorological science teaching at the IPC throughout the author's teaching and research career, after thirty years of experience in the aforementioned institution. It concludes with the aim of contributing to the training of future teachers of Environmental Education in the face of today's dynamic challenges. 


\section{INTRODUCCIÓN}

La meteorología y la climatología, disciplinas que se ocupan respectivamente del estudio físico y estadístico de las condiciones atmosféricas, son ciencias que aportan información de vital importancia tanto para reguardar a los habitantes del planeta de las fuerzas destructivas que pueden desencadenarse en la envoltura gaseosa de la Tierra, como para permitir el aprovechamiento de los recursos naturales renovables que ofrece la dinámica atmosférica. De allí que los servicios meteorológicos se hayan convertido en instituciones fundamentales para el normal desenvolvimiento de las sociedades, tanto en sus actividades cotidianas como aquellas relacionadas con la agricultura, la industria, los problemas de contaminación del aire y del agua, el aprovechamiento de los recursos hídricos y de las fuentes de energías renovables como las que pueden obtenerse del viento y de la radiación solar, de tanta relevancia por tratarse de energías prácticamente inagotables y cuya explotación genera impactos ambientales muy bajos en comparación con el uso de los combustibles fósiles.

A escala global, las ciencias meteorológicas son, desde hace ya algunas décadas, las llamadas a diagnosticar, prevenir $\mathrm{y}$, en lo posible ayudar a la mitigación, del grave problema que representa el cambio climático debido al aumento de los gases de efecto invernadero, por causa de las actividades antrópicas.

Desde la década de los años setenta del siglo pasado, las disciplinas atmosféricas se han considerado en sus interrelaciones con las ciencias que se ocupan de las restantes componentes del sistema Tierra -litosfera, hidrósfera, criosfera y biosferay por tanto enfocadas como todas ellas desde la óptica de la pedagogía del medio ambiente (Flores, 2013).

La idea fundamental de esta pedagogía fue lanzada en la conferencia de Estocolmo, en 1972, sobre cuyas bases posteriormente la UNESCO fijó las grandes líneas de un amplio programa de cooperación internacional. El mismo año, del coloquio de Aix en Provence emanaría el concepto de medio ambiente. Los objetivos de la pedagogía del medio ambiente serían fijados pocos años después en el coloquio de Belgrado, en 1975, y su naturaleza precisada en la conferencia de Tbilisi, en octubre de 1977 (Giolitto, 1984). La Organización Meteorológica Mundial, como agencia especializada de la Organización de Naciones Unidas, se acogió en los años sucesivos a esos principios e hizo de la educación y la enseñanza uno de sus programas básicos, expresado con el objetivo de "alentar la investigación y la enseñanza de la meteorología" (OMM, 1986, p. 15).

Sobre la base de lo antes expuesto, en este trabajo se persiguió el propósito de presentar un recuento del desarrollo de los cursos de meteorología en el IPC, a lo largo de tres décadas (1971-2001), años durante los cuales el autor tuvo la oportunidad de participar en la elaboración de varios programas y de contribuir a formar docentes de educación media, quienes posteriormente desarrollaron sus carreras pedagógicas en todo el territorio 
nacional. Estos programas contribuyeron también a formar profesionales que luego asumieron las responsabilidades de enseñar éstas y otras disciplinas conexas, no sólo en la Universidad Pedagógica Experimental Libertador (UPEL), sin en otras instituciones de Educación Superior en Venezuela y, tras la diáspora que se inició hace unos años, en diferentes países de Latinoamérica, entre los que destacan Perú, Ecuador y Colombia, además de Estados Unidos de Norteamérica y Europa.

El objetivo general del artículo es presentar una visión retrospectiva, basada en la experiencia del autor, con la finalidad de formular algunas reflexiones y recomendaciones que puedan servir para mejorar la calidad de la enseñanza de estas componentes de la Educación Ambiental, con la esperanza de que puedan servir a los futuros estudiantes y docentes para enfrentar los grandes retos que deberá asumir la Educación, ante las amenazas que plantean problemas como el calentamiento global y las graves crisis ambientales que ya están comenzando a manifestarse en muchos países del mundo, tales como la carestía de recursos hídricos, el colapso de la producción de energía hidroeléctrica, los episodios críticos de contaminación del aire, los incendios forestales incontrolables y la alarmante disminución de la biodiversidad en extensos ecosistemas del planeta, especialmente en la zona intertropical, en la que se localiza el territorio venezolano.

\section{Algunos antecedentes}

El Instituto Pedagógico de Caracas fue fundado en 1936 con el propósito de formar docentes para la Educación Media. Diez años después, en dicha institución de Educación Superior se fundó el Departamento de Geografía Historia, cuyo plan de estudios no incluía ninguna asignatura dedicada específicamente a la meteorología, por lo que los contenidos generales relacionados con la dinámica atmosférica estaban incorporados al programa del curso denominado Geografía Física.

En 1969 se implantó un nuevo plan de estudios en el Departamento de Geografía e Historia, en el cual se incluyó un curso denominado Introducción a la Climatología, cuyos contenidos correspondían a un programa de climatología física, con una estructura y contenidos similares a la clásica obra de Landsberg (1958). Este curso fue diseñado e impartido durante diez años por el profesor Antonio Goldbrunner, cofundador del Servicio de Meteorología de la Fuerza Aérea Venezolana (SEMETFAV) y para la época Representante Permanente de Venezuela ante la Organización Meteorológica Mundial (OMM). Durante diecinueve años dicho curso fue el único ofertado en el área de atmósfera a los estudiantes de pregrado.

A partir de 1971, esta asignatura se incluyó como obligatoria para los alumnos del recién fundado Departamento de Ciencias de la Tierra y como electiva para los estudiantes del Departamento de Biología y Química.

\section{Los nuevos cursos}

En 1988, se consolidó la Cátedra de Hidrometeorología en el Departamento de Ciencias de la Tierra del Instituto Pedagógico de Caracas, el cual se había 
integrado a la Universidad Pedagógica Experimental Libertador (UPEL) en 1983. En dicha Cátedra, el autor de este trabajo tuvo la oportunidad de diseñar e implantar tres nuevos cursos en el área de atmósfera, integrados al plan de estudios del mencionado Departamento. Estos cursos se denominaron Meteorología General y Climatología Tropical y de Venezuela, ambos de carácter obligatorio, además de un Seminario electivo para profundizar en la investigación sobre aspectos específicos de la climatología del territorio venezolano.

Cabe destacar que la Cátedra de Hidrometeorología administraba también las asignaturas de Hidrología y Oceanografía (Hidrósfera I/Hidrósfera II). A continuación se describen los cursos creados en 1988 para el plan de estudios del Departamento de Ciencias de la Tierra.

\section{Meteorología General}

Este curso de nivel introductorio contemplaba tanto horas teóricas como prácticas de laboratorio y actividades de observación en el campo (estaciones meteorológicas). El enfoque se concebía integral, ya que propendía a destacar las principales relaciones entre los distintos elementos meteorológicos, a la vez que las de éstos con los factores astronómicos y geográficos que los condicionan y modifican.

El objetivo general de Meteorología General fue proporcionar a los alumnos el marco teórico para abordar el estudio científico de la dinámica atmosférica. También se planteaba la discusión de la evolución geológica de la atmósfera y de su composición química, así como de su estructura térmica, a la vez que de la naturaleza física de los elementos que conforman el tiempo meteorológico y de los principales métodos e instrumentos de observación, enmarcados en su organización mundial como parte de los programas básicos de la OMM (Sistema Mundial de Observación Meteorológica, Sistema Mundial de Análisis de Datos y Sistema Mundial de Telecomunicaciones Meteorológicas).

El programa también incluía aspectos generales sobre los principales problemas atmosféricos globales, como el calentamiento global, la destrucción del ozono estratosférico, el fenómeno de El Niño (ENSO) y la oscilación noratlántica.

Conviene destacar que durante el desarrollo del programa, de duración semestral como todos los restantes cursos, se enfatizaba en las fuentes de los datos meteorológicos, con el propósito de sentar las bases para los cursos subsiguientes, dedicados a la investigación de las condiciones climatológicas del territorio venezolano.

\section{Climatología Tropical y de Venezuela}

Como muchos países localizados en la zona intertropical, Venezuela encuentra en su dinámica atmosférica una dualidad de condiciones, en cierto modo de signos opuestos. Por una parte, las características de ciertos patrones pluviométricos interanuales, con alta variabilidad tanto espacial como temporal, pueden ser causa de severas sequías, como las que se presentaron en 1983-1984, asociadas a un intenso episodio de El Niño, o de graves inundaciones y deslizamientos, como los 
que desencadenó el paso de la tormenta tropical Bret, en agosto de 1993.

Estas condiciones extremas son de difícil pronóstico, en parte debido a la complejidad de la dinámica atmosférica intertropical, aunado a la deficiente cobertura de la red de observación meteorológica tanto de superficie como de altura, a la vez que a la carencia de series temporales de registros meteorológicos de suficiente extensión, problemas que se presentan, aún en la actualidad, en vastas áreas del país.

Por otra parte, las condiciones climatológicas del territorio venezolano representan también una importante fuente de recursos naturales, insuficientemente conocidos aún, entre las cuales pueden destacarse la uniformidad térmica y las temperaturas medias atenuadas de las regiones montañosas, condiciones ambientales favorables para el emplazamiento de centros poblados y para la planificación de actividades agrícolas; los inmensos volúmenes de agua precipitados en las cuencas de los ríos guayanesas, que le confieren un alto potencial hidroeléctrico, así como los elevados montos de insolación y de radiación solar que se registran en extensas áreas, sobre todo de la región septentrional del país, radiación que podría ser aprovechada como fuente alterna de energía, por medio de sistemas de celdas fotovoltaicas, sin descartar las potencialidades que representa la energía eólica.

Lo anteriormente expuesto fundamenta la necesidad de que los docentes de Educación Media de las especialidades de Geografía y de Ciencias de la Tierra se familiaricen con las condiciones climatológicas del país en el que van a desarrollar su carrera pedagógica, a fin de adquirir un conocimiento completo de su geografía física como fundamentos de la educación ambiental y del ordenamiento territorial.

Con base en lo expuesto, el programa de la asignatura incluía conceptos y técnicas de análisis climatológico, a la vez que el análisis tanto estadístico como cartográfico de elementos climatológicos como la temperatura, la insolación, la radiación solar, la nubosidad, la dirección y velocidad del viento, la precipitación y la evaporación.

Los factores que determinan $y$ modifican temporal y espacialmente los mencionados elementos son igualmente objeto de estudio, a la vez que se analizan las principales diferencias entre las condiciones intertropicales y extratropicales, con el fin de resaltar la conveniencia de implementar técnicas de observación acordes con las peculiares características de las condiciones climatológicas del territorio venezolano.

\section{El seminario electivo en climatología de Venezuela}

Dada la corta tradición de los estudios referidos a las condiciones climatológicas regionales y locales del territorio venezolano, con escasas excepciones es poco lo que se ha investigado a estas escalas, realidad que persiste en el presente. Particularmente en lo tocante a la influencia de la topografía sobre los regímenes diarios de precipitación, nubosidad, temperatura y viento, todavía existen muchos interesantes aspectos por investigar, lo cual permite desarrollar este 
tipo de estudios a nivel de pregrado, aun sin contar con mayores recursos ni técnicas de investigación, de aquí que estos estudios elementales se conviertan en una valiosa estrategia para iniciar a los alumnos en las actividades de investigación y para profundizar en la enseñanza de los problemas meteorológicos, sin desconocer que éstos revisten una gran complejidad, dada la multiplicidad de factores que intervienen en su dinámica, lo cual hace particularmente complicada su enseñanza, especialmente en los países en los que estas disciplinas han alcanzado menor desarrollo y cuentan con limitados recursos, tal como lo señalan estudios ya clásicos referidos a la formación profesional en meteorología (Puigcerver, 1972; Van Mieghem, 1963).

A través de este seminario, optativo como se ha señalado, se investigaron aspectos particulares de algunos regímenes pluviométricos en regiones montañosas, como la cordillera de la costa (Foghin-Pillin y Millán, 2007); problemas relacionados con lluvias de extraordinaria magnitud y su impacto en algunas regiones venezolanas (FoghinPillin, 2001); características de algunos sistemas de vientos locales, tipo brisas de mar-tierra y de valle-montaña (FoghinPillin y Reyes, 1999; Reyes y FoghinPillin, 1997), además de problemas relacionados con contaminación atmosférica a escala local.

Entre las actividades de este seminario se incluían frecuentes trabajos de campo, a distintas regiones de Venezuela, con el propósito de que estudiantes y docentes conocieran las características geográficas de las áreas en estudio.

Los resultados de las investigaciones realizadas en el Seminario usualmente se presentaban tanto en la Jornada Anual de Investigación del IPC, como en la convención anual de la Asociación Venezolana para el Avance de la Ciencia (ASOVAC), a la vez que dieron base para algunas publicaciones, como las que se citaron más arriba.

\section{El apoyo de los organismos públicos relacionados con meteorología}

A lo largo de más de tres décadas, para el desarrollo de los cursos del área de meteorología el IPC recibió el apoyo de varios organismos públicos relacionados con el medio ambiente y específicamente la meteorología. En este sentido cabe destacar las relaciones establecidas entre el IPC y SEMETFAV, las cuales comienzan con el ingreso del profesor Goldbrunner al personal docente del IPC. Goldbrunner, cofundador de SEMETFAV, como se indicó, estuvo al frente, por muchos años, de la Asesoría Técnica de dicho organismo.

A partir de la incorporación de Goldbrunner al IPC, SEMETFAV organizó visitas periódicas de los estudiantes y docentes de las asignaturas antes mencionadas, durante las cuales se efectuaban prácticas de observación especialmente en la estación meteorológica sinóptica de Maracay-Boca de Río, dictándose además charlas sobre predicción meteorológica, satélites meteorológicos, etc.

Por otra parte, la Jefatura del mencionado Servicio facilitó 
permanentemente material para las actividades prácticas (laboratorio), como bandas registradoras, mapas sinópticos, imágenes satelitales, registros climatológicos y algunos instrumentos meteorológicos (actinógrafo, heliógrafo, termógrafo, barógrafo, barómetro, evaporígrafo, pluviógrafos, termómetros, etc.), que se usaban para la instrucción de los alumnos del curso básico.

Dentro de la amplia colaboración recibida por parte de SEMETFAV, merecen especial mención las pasantías facilitadas, en distintas oportunidades, a docentes de las asignaturas arriba mencionadas. Estas pasantías, realizadas por algunos profesores a finales de la década de 1970 e inicios de la década de 1980, incluyeron experiencias directas en todas las secciones y departamentos de SEMETFAV.

De igual modo, cabe destacar el apoyo recibido para el desarrollo de las asignaturas citadas, con la organización de ciclos de conferencias sobre temas atmosféricos, dictadas por los meteorólogos de SEMETFAV, encabezados por el profesor Goldbrunner y coordinados por el autor de este trabajo. Dichos ciclos de charla produjeron además una serie de materiales impresos que aún en el presente pueden consultarse en las bibliotecas de SEMETFAV y del IPC (Caldera, 1976; Goldbrunner, 1976; Pacheco, 1976).

De esta manera, en alguna medida se cumplió, durante el lapso aquí reseñado, la aspiración expresada por Puigcerver en el trabajo antes citado, en el que expresaba la conveniencia de la cooperación entre los servicios meteorológicos y las universidades, mediante una conexión efectiva y práctica que pudiese conducir a la activación o revitalización de la investigación y al mismo tiempo contribuyese a dar visibilidad pública a las actividades de los servicios meteorológicos y a divulgar su importancia, de tal forma que ambas instituciones resultaran favorecidas (Puigcerver, 1972).

En el caso concreto aquí descrito, la ventaja adicional que presentaba esta relación para el servicio meteorológico nacional, se fundaba en que los estudiantes universitarios se convertían en docentes que podían divulgar los beneficios de la meteorología en toda la extensión del territorio nacional donde desarrollarían su carrera pedagógica.

Entre los organismos públicos que colaboraron activamente con el desarrollo de las actividades docentes en el área de meteorología en el IPC, también hay que citar al Observatorio Cagigal, adscrito a la Comandancia General de la Marina y al Departamento de Hidrometeorología de la Facultad de Ingeniería de la Universidad Central de Venezuela (UCV), instituciones que también con frecuencia facilitaban el acceso a sus estaciones meteorológicas, en la ciudad de Caracas, para el desarrollo de actividades prácticas con los alumnos del IPC. Es importante resaltar que el Departamento de Hidrometeorología (UCV) tiene el rango de Centro Regional de Formación Profesional de la OMM.

En este punto no puede dejar de mencionarse también la valiosa cooperación recibida por parte de la Oficina de Educación de la Organización Meteorológica Mundial (OMM), en la 
persona de su entonces director, el doctor Gustavo Necco, quien hizo llegar al IPC importantes materiales publicados por aquella agencia especializada de la Organización de Naciones Unidas (OMM, 1990a, OMM, 1990b; OMM, 1992; OMM, 1996). Aparte del valor monetario de estas publicaciones, por completo fuera de las posibilidades del Instituto Pedagógico, hay que resaltar el hecho de que los voluminosos materiales eran enviados desde Suiza a Venezuela por correo ordinario.

\section{Índices de cursantes, repetición y niveles de rendimiento}

A lo largo de tres décadas, el número total de cursantes, considerando las distintas asignaturas reseñadas, alcanzó a unos dos mil aproximadamente, con un mayor porcentaje de estudiantes del sexo femenino.

Desde principios de la década de 1970 los cursos fueron de régimen semestral, con secciones teóricas y grupos de práctica (laboratorio) en los cuales se admitía un máximo de 15 alumnos. Cada curso se dictaba en cinco horas semanales, divididas en dos horas teóricas y tres prácticas, además de las horas dedicadas a las salidas de campo y trabajo en estaciones meteorológicas, cuya duración variaba de acuerdo a circunstancias como las posibilidades de transporte y las condiciones meteorológicas, según la época del año. El número de unidades de créditos era de tres por cada asignatura, sobre un total de 120 unidades que los estudiantes debían aprobar como requisito de grado.
Es importante señalar que en el plan de estudios del Departamento de Ciencias de la Tierra los requisitos de grado incluían un trabajo final, a modo de tesina, en el cual algunos graduandos desarrollaban proyectos que habían iniciado en el Seminario de Climatología de Venezuela, además de seminarios en otras áreas de las geociencias, desde luego, lo cual era optativo de cada cursante que a los efectos recibía la orientación de un tutor. Estos trabajos de grado, como ya se señaló, fueron la base de algunas publicaciones posteriores y, en algunos casos, signaron la disposición del estudiante graduado para realizar sus estudios de maestría y doctorado.

La escala de calificación era de nueve puntos con aprobación a partir de cinco puntos. Es pertinente resaltar que los índices de rendimiento, en promedio, superaban escasamente el nivel de aprobación y el índice de repetición era, particularmente en el curso de Introducción a la Climatología, superior al $30 \%$, lo cual siempre se consideró alto y se atribuyó al escaso dominio que mostraban los cursantes en los conocimientos que deberían haber proporcionado las asignaturas exigidas como prelación, las cuales eran Geografía General I y Matemática General, para los cursantes del Departamento de Geografía e Historia, e Introducción a las Ciencias de la Tierra y Física I para los del Departamento de Ciencias de la Tierra. Estos problemas se atribuyeron a la falta de coordinación entre los cursos de prelación y los cursos de la especialidad, además de ciertas deficiencias básicas en lectura, 
comprensión de textos y redacción, que muchos cursantes arrastraban desde Educación Media.

En el caso de los Seminarios, el nivel de repetición prácticamente se reducía a cero y los índices de calificaciones eran considerablemente mayores, lo que podría explicarse por el carácter optativo del Seminario, lo cual garantizaba la motivación del estudiante por estos estudios, además de que operaba una cierta selección espontánea que dirigía hacia el Seminario a los estudiantes con los mayores niveles de rendimiento académico en las asignaturas previas.

Otros factores adversos, de no menor importancia, que sin duda incidían en el rendimiento académico de los estudiantes, se relacionaban con la escasa bibliografía actualizada, en lengua española, que podían disponer en las bibliotecas del IPC; no debe olvidarse que hasta finales del siglo XX el recurso de Internet no estaba generalizado ni disponible en el país, ni siquiera para el personal docente. Se trabajaba, en consecuencia, con algunos libros clásicos editados en español en las décadas de 1960-1970 (Hernández, 1968; Donn, 1978; Durand-Dastès, 1969; Miller, 1975; Petterssen, 1976). Para los contenidos de climatología de Venezuela, los libros de Vila (1960) y Goldbrunner (1958; 1976) fueron materiales de consulta ampliamente utilizados.

La escasa bibliografía disponible en inglés, para la época, tampoco era de fácil acceso para los estudiantes, sin excluir de esta limitación a muchos docentes, debido al deficiente dominio de esa lengua, inclusive a nivel instrumental.

\section{Principales logros}

Pese a las dificultades que se confrontaron en el IPC a lo largo de las tres décadas aquí reseñadas, para la implantación y desarrollo de los programas relacionados con las disciplinas meteorológicas, pueden enumerarse algunos logros significativos para la educación y la investigación en el área de la Geografía Física y de las geociencias en Venezuela.

El primero de dichos logros ya se señaló y consiste en haber podido hacer llegar hasta los más apartados lugares de la geografía nacional, las nociones relacionadas con las disciplinas atmosféricas como parte de las ciencias del medio ambiente $\mathrm{y}$, por ende, como componentes fundamentales de la Educación Ambiental, es decir, se contribuyó significativamente a lo que se ha Ilamado la pedagogía del medio ambiente (Giolitto, 1984), dado que los cursos descritos no sólo contemplaban entre sus propósitos generales la transmisión de contenidos meteorológicos y climatológicos, sino que incluían objetivos específicos en los que se contemplaban las estrategias didácticas para impartir dichos conocimientos en el nivel de Educación Media y se planteaba un enfoque holístico de las disciplinas meteorológicas, destacándose las relaciones con las condiciones geográficas regionales (geomorfología, suelos, vegetación, cultivos, tipos de vivienda) y, en el ámbito de las políticas públicas, con el ordenamiento territorial.

Cabe destacar, así mismo, las permanentes discusiones en las sesiones de clase a partir de los conocimientos 
previos que podían traer los estudiantes desde sus respectivas regiones de procedencia, dándose de esta forma un enfoque constructivista a la enseñanza (Ausubel, Novak y Hanesian, 1983; Vygostki, 1995).

Las carencias de material de consulta para los estudiantes, antes aludidas, eran más acentuadas en los aspectos prácticos, por lo que en 1990, como parte de la Colección Difusión, creada por iniciativa del entonces Jefe del Departamento de Ciencias de la Tierra profesor Carlos Suárez Ruiz, se publicó un folleto ilustrado de 51 páginas en el cual se exponían los principales aspectos de la observación meteorológica (Foghin-Pillin, 1990). Editado en forma más bien modesta y con un reducido tiraje, este trabajo tuvo sin embargo buena acogida también por parte de la Escuela Técnica de la Fuerza Aérea Venezolana, en la cual se formaba el personal técnico que requería SEMETFAV, lo cual pone de relieve, una vez más, la importancia de la cooperación entre los servicios meteorológicos y las universidades.

Las actividades desarrolladas a través de los cursos que se han comentado, permitieron la participación del IPC en la Fourth International Conference on School and Popular Meteorological and Oceanographic Education, que se efectuó en la ciudad escocesa de Edimburgo (Reino Unido), del 22 al 26 de julio de 1996, patrocinada por la Organización Meteorológica Mundial, la American Meteorological Society y la Royal Meteorological Society, evento en el que se presentó una ponencia sobre la enseñanza de las ciencias meteorológicas en el IPC (Foghin-Pillin, 1996).

Con la experiencia acumulada y ante la persistente falta de un texto que recogiera las principales características del territorio venezolano, en lo tocante a sus condiciones meteorológicas, tras la jubilación de quien escribe se publicó un libro de 150 páginas (Foghin-Pillin, 2002), que contribuyó a satisfacer, en alguna medida, aquellos requerimientos. A pesar de no haber podido actualizarse y reeditarse, se puede comprobar que la obra aparece citada en diferentes investigaciones, aun en otros países.

Finalmente, como producto de las experiencias acumuladas a lo largo de tres décadas, en 2004 se publicó un libro sobre la vida y obra del pedagogo y geógrafo Pablo Vila (Foghin-Pillin, 2004), fundador de los modernos estudios geográficos en Venezuela; en la tercera parte de dicho libro, basada en un trabajo de ascenso presentado en 1992 por el autor, se analizan detalladamente y actualizan los quince capítulos que el renombrado educador catalán dedicara a la climatología de Venezuela en su ya citada obra (Vila, 1960).

\section{Los recursos de Internet en la enseñanza de las ciencias meteorológicas}

A comienzo de la década de 1990 comenzó a difundirse en Venezuela el uso de Internet y se hicieron accesibles, desde computadoras personales, las plataformas electrónicas que desplegaban información meteorológica diaria, principalmente mapas sinópticos e imágenes satelitales a escala hemisférica, que comprendían 
también el territorio venezolano. Del mismo modo se hicieron crecientemente accesibles las bases de datos climatológicos de muchos de los países miembros de la OMM.

La disponibilidad de los citados materiales significó un enorme avance para la enseñanza de las disciplinas en cuestión y para la investigación que podía desde entonces realizarse desde las aulas y laboratorios, con las únicas limitaciones que podían suponer la velocidad de acceso a la información on-line y los medios audiovisuales para el uso de la información ante grupos de estudiantes. En Venezuela, todas esas posibilidades experimentaron un período de rápida expansión desde aquellos años iniciales hasta, podría decirse, los primeros dos lustros del presente siglo.

Desde los primeros años de la aparición de Internet como servicio público, los docentes de ciencias de la atmósfera se percataron del inmenso potencial didáctico que ofrecía las nuevas tecnologías. Encuestas realizadas tras los primeros años de uso de estos recursos en la enseñanza de la meteorología, evidenciaron el desarrollo más rápido de una percepción a escala global de los fenómenos meteorológicos, facilidad en la adquisición de la información, disponibilidad de una amplia variedad de materiales e información, posibilidad de comunicación con expertos de todo el mundo, oferta de gran variedad de actividades en convenciones, congresos, etc., posibilidad de permanente actualización, disponibilidad de información para los diferentes niveles de los planes de estudio y flexibilización de las posibilidades del estudiante para avanzar a su propio ritmo (Donert, 1996; Duncan, 1996; Pascuali, 1998; Scott, Alexander, Collins y Smith, 1997).

De esta manera, todas las limitaciones que habían gravado sobre la experiencia docente de tres décadas, referidas en este trabajo, quedaban, al menos teóricamente, superadas y se planteaba la perspectiva de que las nuevas tecnologías se convirtieran "en el instrumento definitivo para la democratización del saber" (Pascuali, 1998, p. 285).

Todas las ventajas enumeradas unas líneas más arriba, quedaron confirmadas por investigaciones más recientes, tras ya varios lustros de empleo didáctico de los recursos de Internet para la enseñanza de la meteorología y disciplinas afines (Gómez, 2016; Gómez, Molina y ReyesLabarta, 2016). Sin embargo, ya para 1998, las estadísticas permitían constatar que Internet se expandió conforme al perfil de la distribución mundial de riqueza, con casi un $82 \%$ de los usuarios en el hemisferio norte (EEUU, Canadá, Europa y Japón y sólo algo más del 18\% en el hemisferio sur (Pascuali, 1998). En ese contexto, la situación de Venezuela probablemente haya evolucionado aún menos satisfactoriamente, sobre todo tras el período de crecimiento antes señalado.

La experiencia personal con el uso de Internet como recurso didáctico en la docencia de las disciplinas relacionadas con la atmósfera, permite enumerar algunos de los conceptos y contenidos, de menor o mayor complejidad, que pueden presentarse a los estudiantes en distinto niveles, pero con igual provecho: sucesión día-noche; duración del día; estacionalidad geoastronómica; calentamiento diferencial 
de las superficies marinas y continentales; mapas sinópticos de superficie y altura; interpretación de imágenes satelitales; el fenómeno de El Niño; el problema de la destrucción del ozono estratosférico; la oscilación noratlántica, entre muchos otros.

Por lo que atañe a las posibilidades de investigación en meteorología, mediante el uso de los recursos de Internet, en particular de las imágenes de los satélites meteorológicos (NASA, 2020), con su aplicación el autor ha podido identificar algunos sistemas nubosos regionales, no descritos anteriormente en la literatura geográfica venezolana (Foghin-Pillin, 2014; 2016). Investigaciones de este género pueden desarrollarse tanto a nivel de pregrado como de postgrado, en los cursos de meteorología que se ofrezcan en cualquier institución de Educación Superior e inclusive en Educación Media, con las adaptaciones de nivel pertinentes.

Finalmente, con relación a estos aspectos resulta importante señalar también que muchas organizaciones relacionadas con la ciencias atmosféricas mantienen actualmente secciones dedicadas específicamente a temas educativos en sus plataformas on-line (American Meteorological Society, 2020; NASA, 2020b; National Hurricane Center, 2020; NOAA, 2020). La OMM (WMO) por su parte, frecuentemente publica en línea materiales relacionados con temas meteorológicos de interés mundial (WMO, 2020).

\section{CONCLUSIONES}

La implantación del llamado Nuevo Diseño Curricular en el IPC y posteriormente de los planes de estudios con la creación de la UPEL, marcan el final de dos etapas en la historia de la primera casa de formación docente venezolana, aunque es forzoso señalar que en sus momentos respectivos los cambios no fueron precedidos por un proceso de evaluación formal sobre las actividades realizadas, los resultados obtenidos en función de los objetivos planteados, el rendimiento académico, la vigencia de los contenidos programáticos y la funcionalidad de los prerrequisitos, por lo cual, el presente trabajo, circunscrito al área de las ciencias meteorológicas, también representa, en cierto modo, una evaluación parcial, formulada desde la óptica de un docente que participó durante cerca de tres décadas en esos procesos, a través de las actividades de enseñanza, investigación y extensión.

Con base en dicha experiencia, puede afirmarse que puede mejorarse en gran medida el rendimiento estudiantil en las asignaturas relacionadas con las disciplinas atmosféricas orientando efectivamente a los alumnos sobre la cantidad de horas de estudio que deben dedicar a cada tema, estableciendo una efectiva coordinación entre los docentes de las materias de la especialidad con los docentes que imparten las asignaturas establecidas como prerrequisitos, así como de las asignaturas instrumentales (Lengua Española, Idiomas Modernos).

La experiencia indica que si bien el número de estudiantes de las secciones teóricas sólo debería estar limitado por las condiciones del espacio físico en el que se desarrollen las clases (aulas, auditorios), para los grupos de prácticas (laboratorio), 
en cambio, quince alumnos parece ser el máximo recomendable si se cuenta con un auxiliar docente (preparador), o diez en caso de que no se disponga de tal apoyo.

Se considera indispensable poner a disposición de los alumnos una biblioteca especializada, a los efectos de que puedan consultar la bibliografía clásica, dado que el conocimiento de la historia de las teorías y técnicas en meteorología permite una comprensión más cabal de los avances realizados para llegar al estado actual del conocimiento en estas ciencias. Desde luego, dichas bibliotecas deberán contar también con eficientes conexiones a Internet y suficientes terminales para satisfacer las demandas de los usuarios.

Es altamente deseable planificar ciclos periódicos de conferencias, dictadas por especialistas de otras instituciones, las cuales han sido señaladas en las páginas precedentes. Del mismo modo, deben reactivarse y reforzarse los nexos con los organismos públicos con competencias en el área de meteorología y las instituciones de Educación Superior deben apoyar a dichos organismos en lo tocante a las funciones de investigación y extensión, incluyendo las relaciones con los medios de comunicación, como ha recomendado la OMM (1987).

Finalmente, se recomienda retomar y llevar a cabo el proyecto de la instalación de una estación meteorológica en cada Instituto Pedagógico que integra la UPEL, en las distintas regiones de Venezuela, a la vez que un observatorio meteorológico principal en la sede administrativa de la Universidad (Catia, Caracas), dada la particular situación topográfica de su emplazamiento, la cual resulta idónea para el estudio de los sistemas de vientos que afectan la depresión de Caracas, el conglomerado urbano más extenso y poblado del país, sujeto a problemas meteorológicos relacionados con su pluviometría y susceptible ante los episodios de contaminación atmosférica.

Considerando la cobertura geográfica de la UPEL, la cual abarca gran parte del territorio nacional, la implementación de la mencionada red de estaciones constituiría un valioso aporte al conocimiento de las condiciones climáticas del país, al tiempo que fortalecería las bases y proporcionaría los instrumentos para extender la educación ambiental hacia los más amplios sectores sociales, dadas las capacidades de los docentes como factores multiplicadores.

\section{REFERENCIAS}

American Meteorological Society: (2020). Education and Careers. Boston: American Meteorological Society. Recuperado de https://www.ametsoc.org/index.cfm/am s/education-careers/

Ausubel, D., Novak, J., y Hanesian, H. (1983). Psicología educativa: un punto de vista cognoscitivo. $2^{a}$. Ed. México: Editorial Trillas

Caldera G., E. A. (1976). Historia de la meteorología en Venezuela y el mundo. Curso de Extensión en Meteorología. Caracas: Instituto Universitario Pedagógico de Caracas/Servicio de Meteorología de la Fuerza Aérea Venezolana

Donert, K. (1996). Using the Internet to assist the teaching of meteorology. Ponencia presentada en The Fourth International Conference on School and Popular Meteorological and 
Oceanographic Education. Preprint volume, p. 52-55. Edinburgo, Gran Bretaña, 22 al 26 de julio de 1996

Donn, W. (1978). Meteorología. Barcelona: Editorial Reverté

Duncan, C. (1996). Mutimedia technology: Can we live with it? Can we live without it? Ponencia presentada en The Fourth International Conference on School and Popular Meteorological and Oceanographic Education. Preprint volume, p. 64-71. Edinburgo, Gran Bretaña, 22 al 26 de julio de 1996

Durand-Dastès, F. (1972). Climatología. Barcelona: Ediciones Ariel

Flores, R. C. (2013). Diálogos entre la pedagogía y la educación ambiental. Revista Educación y Desarrollo Social, 7(1), 95-107. Recuperado de https://revistas.unimilitar.edu.co/index.p $\mathrm{hp} / \mathrm{reds} /$ article/view/737

Foghin-Pillin, S. (1990). Introducción a la observación meteorológica. Caracas: Universidad Pedagógica Experimental Libertador

Foghin-Pillin, S. $\quad 1996 . \quad$ Teaching meteorology for 25 years at the IPCUPEL: a Venezuelan experience. Ponencia presentada en The Fourth International Conference on School and Popular Meteorological and Oceanographic Education. Preprint volume, p. 89-92. Edinburgo, Gran Bretaña, 22 al 26 de julio de 1996

Foghin-Pillin, S. y Reyes, V. M. (1999). Descripción de algunas circulaciones periódicas locales en las costas venezolanas. Revista Terra, 15(24), 79-98

Foghin-Pillin, S. (2001). Algunas consideraciones meteorológicas acerca de la catástrofe del estado Vargas en diciembre de 1999. Aula y Ambiente, 1(1), 93-98

Foghin-Pillin, S. (2014). Evidencias de dos sistemas de circulación a meso-escala en la península de Paraguaná,
Venezuela. Revista Catalana de Geografía, $\quad 48,1-7$ http://www.rcg.cat/articles.php?id $=282$ Foghin-Pillin, S. (2016). Evidencias de la penetración de brisas de mar en la depresión del río Unare y Llanos de Anzoátegui. Revista de Investigación (UPEL-IPC), 40 (87): 39-62

Foghin-Pillin, S. y Millán, Z. (2007). Observaciones pluviométricas en San Antonio de los Altos (Estado Miranda) durante los meses de enero a agosto de 2007. Sapiens. Revista Universitaria de Investigación, 8(2), 187-195. Recuperado de https://www.redalyc.org/pdf/410/4108 0212.pdf

Giolitto, P. (1984). Pedagogía del medio ambiente. Barcelona: Editorial Herder

Goldbrunner, A. W. (1960). Las causas meteorológicas de las lluvias de extraordinaria magnitud en Venezuela. Caracas: Ministerio de Obras Públicas

Goldbrunner, A. W. (1976). El clima de Venezuela y su clasificación. Curso de Extensión en Meteorología. Caracas: Instituto Universitario Pedagógico de Caracas/Servicio de Meteorología de la Fuerza Aérea Venezolana

Gómez T., I.M. (2016) La didáctica de la Geografía y las TIC: nuevas metodologías de enseñanza y aprendizaje. En J. D. Álvarez Teruel, M. T. Tortosa Ybáñez, N. Pellín Buades, (Eds.), Investigaciones metodológicas en docencia universitaria: resultados de investigación (pp. 839- 850). Alicante: Universidad de Alicante

Gómez D., I., Molina P., S. y ReyesLabarta, J. A. (2016). Aplicación de una metodología de enseñanzaaprendizaje en Meteorología a través de herramientas de software libre y datos de modelización numérica. En Tecnología, innovación e investigación en los procesos de enseñanzaaprendizaje. Roig-Vila, R. (Edit.), p. 
2078-2087. Barcelona: Ediciones Octaedro

Hernández Y., S. (1968). Meteorología y Oceanografía. Barcelona: Editorial Cadí

Landsberg, H. (1958). Physical Climatology. DuBois, Pennsylvania: Gray Printing

Miller, A. (1975). Climatología. Barcelona: Ediciones Omega

NASA. (2020). Interactive Global Geostationary Weather Satellite Images. Huntsville, Alabama: NASA. Recuperado de https://weather.msfc.nasa.gov/GOES/

National Hurricane Center. (2020). Educational resources. Miami: National Hurricane Center. Recuperado de https://www.nhc.noaa.gov/outreach/

NOAA. (2020). Index of/ tafb. Miami: National Hurricane Center. Recuperado de https://www.nhc.noaa.gov/tafb/

OMM. (1987). La meteorología y los medios de comunicación. Ginebra: OMM

OMM. (1990a). Guía de instrumentos y métodos de observación meteorológicos. Ginebra: Organización Meteorológica Mundial

OMM. (1990b). Guía de prácticas climatológicas. Ginebra: Organización Meteorológica Mundial

OMM. (1992). Vocabulario Meteorológico Internacional. Ginebra: Organización Meteorológica Mundial

OMM. (1996). Climatological Normals (CLINO) for the period 1961-1990. Ginebra: Organización Meteorológica Mundial

Pacheco, P. P. (1976). Características de las precipitaciones en Venezuela. Curso de Extensión en Meteorología.
Caracas: Instituto Universitario Pedagógico de Caracas/Servicio de Meteorología de la Fuerza Aérea Venezolana

Pascuali, A. (1998). Bienvenido Global Village. Caracas: Editorial Monte Ávila

Petterssen, S. (1976). Introducción a la meteorología. Madrid: Espasa Calpe

Puigcerver Z., M. (1972). Algunas consideraciones acerca de la enseñanza de la meteorología. Madrid: Servicio Meteorológico Nacional

Reyes, V. M. y Foghin-Pillin, S. (1997). Descripción del régimen eólico diario 1971-1980 en la estación de Santa Elena de Uairén. Ponencia V Jornada Anual de Investigación (p. 27). Instituto Pedagógico de Caracas, Universidad Pedagógica Experimental Libertador

Scott, J., Alexander, M., Collins, J, y Smith, C. (1997) Interactive visualitation of climate data on the WWW. Bulletin of the American Meteorological Society, 78(9), 1985-1989

Van Mieghem, J. (1963). The problem of the professional training of meteorological personnel of all grades in the less-developed countries. Geneva: World Meteorological Organization

Vygotski, L. (1995). Pensamiento y Lenguaje. Buenos Aires: Ediciones Fausto

Vila, P. (1960). Geografía de Venezuela T1. Caracas: Ministerio de Educación

WMO. (2020). Count every drop. Every drop counts. Geneva: World Meteorological Organization. Recuperado de https://worldmetday.wmo.int/en/conf 